\title{
Ornithine cyclodeaminase-based proline production by Corynebacterium glutamicum
}

\author{
Jaide Vold Korgaard Jensen and Volker Fritz Wendisch*
}

\begin{abstract}
Background: The soil bacterium Corynebacterium glutamicum, best known for its glutamate producing ability, is suitable as a producer of a variety of bioproducts. Glutamate is the precursor of the amino acid proline. Proline biosynthesis typically involves three enzymes and a spontaneous cyclisation reaction. Alternatively, proline can be synthesised from ornithine, an intermediate of arginine biosynthesis. The direct conversion of ornithine to proline is catalysed by ornithine cyclodeaminase. An ornithine overproducing platform strain with deletions of $\arg R$ and $\arg F$ (ORN1) has been employed for production of derived compounds such as putrescine. By heterologous expression of ocd this platform strain can be engineered further for proline production.
\end{abstract}

Results: Plasmid-based expression of ocd encoding the putative ornithine cyclodeaminase of C. glutamicum did not result in detectable proline accumulation in the culture medium. However, plasmid-based expression of ocd from Pseudomonas putida resulted in proline production with yields up to $0.31 \pm 0.01 \mathrm{~g}$ proline $/ \mathrm{g}$ glucose. Overexpression of the gene encoding a feedback-alleviated $\mathrm{N}$-acetylglutamate kinase further increased proline production to $0.36 \pm$ $0.01 \mathrm{~g} / \mathrm{g}$. In addition, feedback-alleviation of $\mathrm{N}$-acetylglutamate kinase entailed growth-coupled production of proline and reduced the accumulation of by-products in the culture medium.

Conclusions: The product spectrum of the platform strain C. glutamicum ORN1 was expanded to include the amino acid L-proline. Upon further development of the ornithine overproducing platform strain, industrial production of amino acids of the glutamate family and derived bioproducts such as diamines might become within reach.

Keywords: Amino Acid, Proline, Corynebacterium Glutamicum, Metabolic Engineering, OCD, ORN1, Ornithine, Ornithine Cyclodeaminase, Platform Strain, Diamine, Putrescine, N-acetylglutamate Kinase

\section{Background}

The workhorse Corynebacterium glutamicum has for decades been used as an amino acid producer. Although, in terms of quantity, the main contributors to the amino acid market are L-lysine and L-glutamate, minor amino acids such as L-proline are also of importance. Proline is predominantly used as an organocatalyst by the chemical industry, as a precursor for compounds with pharmaceutical and cosmetic applications, and as a feed additive [1-3]. The natural functions of proline in prokaryotic and eukaryotic cells have been reported to be as an osmolyte, a potential virulence factor for some pathogenic bacteria, and a source of carbon, nitrogen, and energy [4]. The amino acid functions as a compatible solute of C. glutamicum and in this respect the organism has

\footnotetext{
* Correspondence: volker.wendisch@uni-bielefeld.de

Genetics of Prokaryotes, Faculty of Biology \& CeBiTec, University of Bielefeld, Universitätsstrasse 25,33615, Bielefeld, Germany
}

been shown to grow at intracellular concentrations of up to $94 \mathrm{~g} / \mathrm{L}$ proline with no determined upper limit [5]. In Escherichia coli the bifunctional enzyme PutA catalyses the two-step oxidation of proline to glutamate with proline dehydrogenase and $\Delta 1$-pyrroline-5-carboxylate dehydrogenase activities: reactions that occur at high proline concentrations [6]. C. glutamicum contains a putative putA gene, but the activity of the encoded enzyme has thus far not been confirmed. It is not clear whether C. glutamicum can utilise proline as a carbon or nitrogen source as contradictory statements about proline utilisation by this bacterium have been published $[7,8]$.

Proline is synthesised from glutamate via three enzymatic and one spontaneous reactions, in most investigated microorganisms $[4,9,10]$. The enzymes of the proline pathway encoded by $\operatorname{proB}$, proA, and proC catalyse the phosphorylation of glutamate followed by reduction to glutamate- $\gamma$-semialdehyde, a spontaneous cyclisation, and 
finally the reduction to proline. An alternative route to proline biosynthesis involves ornithine cyclodeaminase (OCD) which catalyses the conversion of ornithine to proline and ammonia with deamination of the $\alpha$-amino group prior to cyclisation (Figure 1). However, only a few organisms such as Clostridium sporogenes, Treponema denticola, Agrobacterium tumefaciens, and Pseudomonas putida have been reported to contain OCD [11-15]. The genome of C. glutamicum contains a putative ocd gene, however, evidence for its function as ornithine cyclodeaminase has not been reported [16].

In C. glutamicum ornithine is an intermediate of arginine biosynthesis and is synthesised from glutamate through five enzymatic steps where the second enzyme, $\mathrm{N}$-acetylglutamate kinase (NAGK), is feedback-inhibited by arginine [17]. It is known that the arginine biosynthetic pathway of C. glutamicum is regulated at the transcriptional level by the repressor ArgR that has been shown to bind upstream regions of $\operatorname{argC}, \operatorname{argB}, \operatorname{argF}$, and $\operatorname{argG}[18]$. Further genetic regulation, although not fully comprehended, is concerted by the acyl-responsive transcriptional regulator FarR and by the potential allosteric inhibition of ornithine acetyltransferase by ornithine $[18,19]$.
An ornithine overproducing C. glutamicum strain $[20,21]$ with deletions of the genes $\operatorname{argR}$ and of $\arg F$, the gene encoding ornithine carbamoyl-transferase, which converts ornithine to citrulline in the arginine biosynthetic pathway, has been constructed. This strain, called ORN1, has the potential of serving as a platform for the production of several industrially relevant bioproducts of the glutamate family; namely ornithine, citrulline, arginine, putrescine, and spermidine. Based on C. glutamicum ORN1, strains PUT1 [22] and PUT21 [23] have been developed for production of 1,4-diaminobutane (putrescine), and ARG1 [20] is an arginine-producing ORN1 derivative. Here, the production of proline based on ORN1 is reported. Heterologously produced ornithine cyclodeaminase from $P$. putida led to a conversion of ornithine to proline and thereby constitutes an expansion of the product palette of the platform strain ORN1.

\section{Results}

\section{Proline utilisation}

To establish if the putative proline degradation system of C. glutamicum had an effect on extracellular accumulation of proline, we first investigated the utilisation

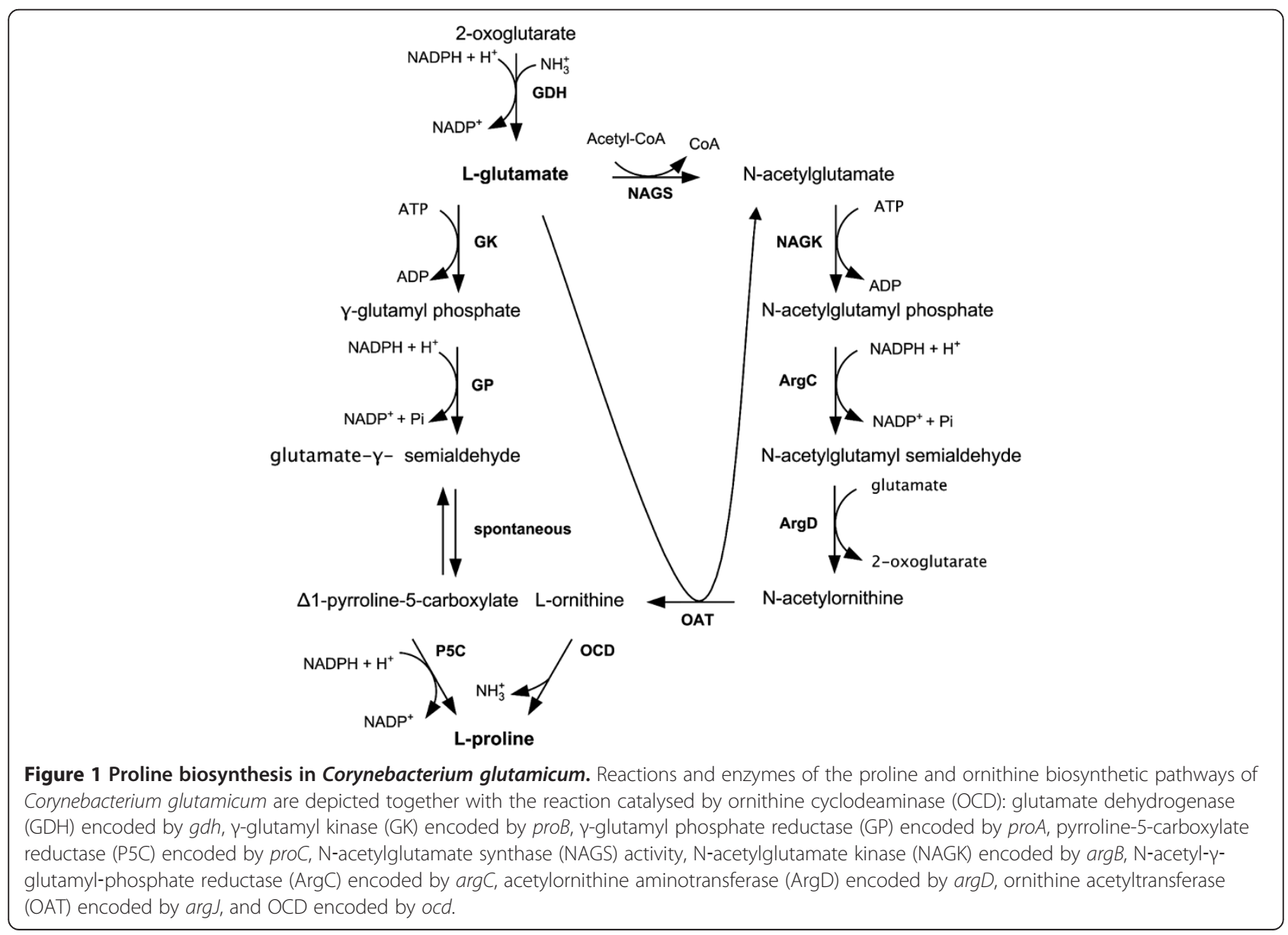


of proline as carbon and nitrogen source. Wild-type C. glutamicum was inoculated to an optical density (OD) of 1 in CGXII minimal medium with $20 \mathrm{~g} / \mathrm{L}$ glucose or a $\mathrm{C}$-equimolar concentration of proline as carbon source and $20 \mathrm{~g} / \mathrm{L}$ ammonium sulfate and $5 \mathrm{~g} / \mathrm{L}$ urea or a $\mathrm{N}$-equimolar concentration of proline as nitrogen source. For cells cultured in medium with proline as carbon source no biomass formation was observed during $48 \mathrm{~h}$ of incubation. An OD of $\sim 30$ could be reached within $10 \mathrm{~h}$ by cultivation in CGXII medium with glucose, ammonium sulfate, and urea. When an $\mathrm{N}$-equimalor proline concentration was used as the sole nitrogen source, the cells were able to duplicate twice in $24 \mathrm{~h}$ within the $48 \mathrm{~h}$ of incubation resulting in a maximum OD of $\sim 3.5$. For comparison an OD of $\sim 1.5$ could be reached when no nitrogen source was added to the medium.

\section{Plasmid-based overexpression of ocd}

The gene encoding the biochemically characterised OCD from P. putida [24] and the putative ocd from C. glutamicum were cloned into the IPTG-inducible expression plasmid pVWEx1 [25]. The resulting plasmids pVWEx1-ocd $C g$ and pVWEx1-ocd ${ }_{P p}$ were transformed into the ornithine producer ORN1 to yield strains JJ002 (ORN1 carrying pVWEx1-ocd ${ }_{C g}$ ) and JJ003 (ORN1 carrying pVWEx1-ocd $\left.d_{P p 1}\right)$. For a presumed more efficient translational termination, the original stop codon TGA of $o c d_{P p}$ was replaced by the more frequently used TAA (JJ004). The specific cyclodeaminase activities in crude extracts of these strains and the control strain JJ001 (ORN1 carrying pVWEx1) were determined and the presence of the overproduced proteins visualised by SDS-PAGE (Figure 2, Table 1). No activity could be observed for crude extracts of JJ001 and JJ002, although the crude extract of JJ002 exhibited a band on an SDS-gel corresponding to the weight of the putative C. glutamicum OCD of $40.96 \mathrm{kDa}$, calculated based on the amino acid sequence (Figure 2). Substitution of the translational stop codon caused an almost 12-fold increased specific activity of $\mathrm{OCD}_{P p}$.

Shake flask fermentations in glucose minimal medium with IPTG were performed with the aforementioned strains. Samples were withdrawn for product quantification by HPLC. Upon glucose depletion, proline could not be detected in the supernatants of strains JJ001 and JJ002, whereas ornithine was produced by both strains (Table 2). By contrast, JJ003 and JJ004 accumulated proline in the supernatant (Figure 3, Table 2). Owing to the feedback inhibition of NAGK by arginine, production of proline was growth decoupled. Strain JJ004 accumulated about three folds more proline than JJ003 indicating that the improved translational termination of $\operatorname{cod}_{P p}$ entailed not only increased OCD activity, but also increased proline production. All strains accumulated ornithine and

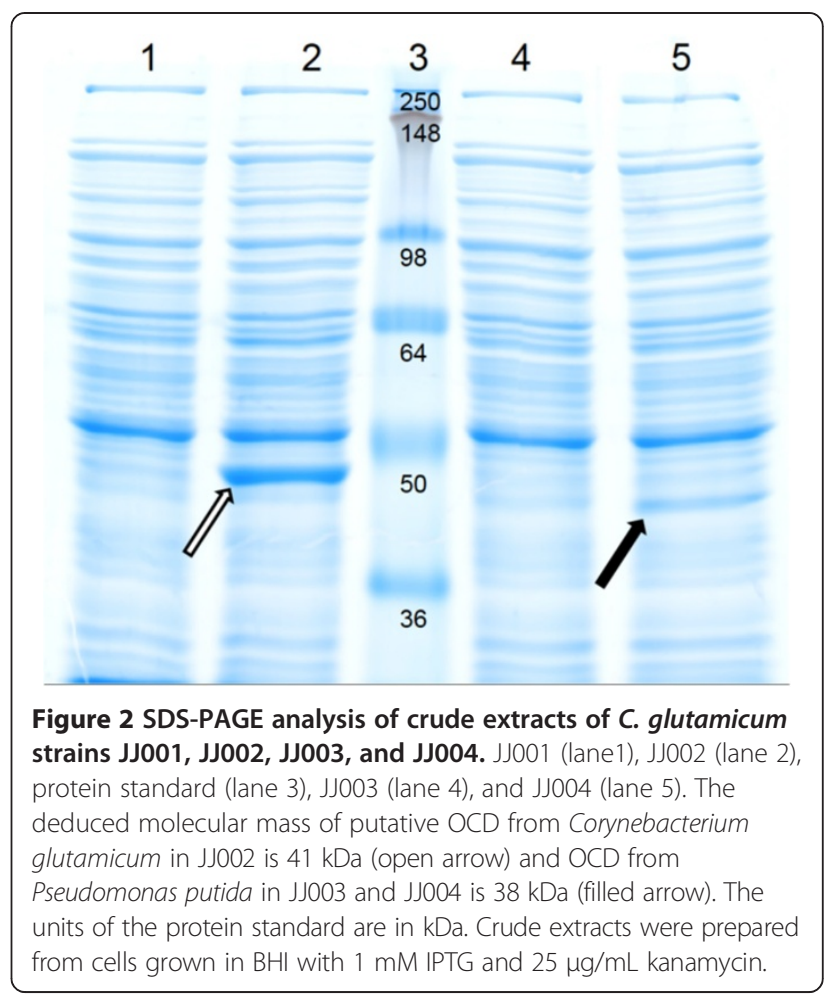

the by-products threonine, alanine, and valine. Furthermore, trace amounts of glutamate (up to $5 \mu \mathrm{m}$ ) could be detected, except for strain JJ003 where $0.24 \pm 0.1 \mathrm{~g} / \mathrm{L}$ was accumulated.

\section{Identification of key medium components for proline production}

As deamination of ornithine by OCD yields ammonia besides proline, and as CGXII contains a high nitrogen concentration, it was tested if the nitrogen content of the medium had an effect on proline formation and if other medium components influenced production. First the effect of the nature of the nitrogen source was tested; here urea, and/or ammonium sulphate or ammonium chloride were selected (Table 3 ). Urea as sole nitrogen source at the concentration tested appeared to be superior for proline overproduction.

Table 1 Ornithine cyclodeaminase activity

\begin{tabular}{ll}
\hline $\begin{array}{l}\text { Crude } \\
\text { extract }\end{array}$ & $\begin{array}{l}\text { Specific activity } \\
(\mu \mathrm{mol} / \mathrm{min} / \mathrm{mg} \text { protein })\end{array}$ \\
\hline$J 001$ & $<4 \cdot 10^{-4}$ \\
$\mathrm{~J} 002$ & $<4 \cdot 10^{-4}$ \\
$\mathrm{~J} 003$ & $0.06 \pm 0.02$ \\
$\mathrm{~J} 004$ & $0.71 \pm 0.09$
\end{tabular}

Crude extracts were obtained by sonication of cells cultured in $\mathrm{BHI}$ medium supplemented with $1 \mathrm{mM}$ IPTG and $25 \mu \mathrm{g} / \mathrm{mL}$ kanamycin. 
Table 2 Accumulation of proline and by-products

\begin{tabular}{llllll}
\hline & $\begin{array}{l}\text { Proline } \\
(\mathbf{g} / \mathbf{L})\end{array}$ & $\begin{array}{l}\text { Ornithine } \\
(\mathbf{g} / \mathbf{L})\end{array}$ & $\begin{array}{l}\text { Threonine } \\
(\mathbf{g} / \mathbf{L})\end{array}$ & $\begin{array}{l}\text { Alanine } \\
(\mathbf{g} / \mathbf{L})\end{array}$ & $\begin{array}{l}\text { Valine } \\
(\mathbf{g} / \mathbf{L})\end{array}$ \\
\hline$J 0001$ & $<10^{-4}$ & $14.8 \pm 0.2$ & $0.3 \pm 0.01$ & $0.7 \pm 0.03$ & $0.7 \pm 0.01$ \\
$J J 002$ & $<10^{-4}$ & $12.1 \pm 0.3$ & $0.3 \pm 0.01$ & $0.5 \pm 0.01$ & $0.5 \pm 0.01$ \\
$J J 003$ & $2.4 \pm 0.1$ & $5.5 \pm 0.3$ & $0.3 \pm 0.01$ & $0.6 \pm 0.01$ & $0.4 \pm 0.01$ \\
$J J 004$ & $10.0 \pm 0.1$ & $2.8 \pm 0.2$ & $0.2 \pm 0.01$ & $0.3 \pm 0.01$ & $0.3 \pm 0.01$ \\
JJ004* & $10.9 \pm 0.3$ & $0.2 \pm 0.1$ & $0.2 \pm 0.01$ & $0.3 \pm 0.01$ & $0.6 \pm 0.01$
\end{tabular}

Titers were obtained from cells grown in CGXII medium with $40 \mathrm{~g} / \mathrm{L}$ glucose, $0.75 \mathrm{mM}$ arginine, $1 \mathrm{mM}$ IPTG, and $25 \mu \mathrm{g} / \mathrm{mL}$ kanamycin. * Titers were obtained from cells grown in CGXIIm medium with $35 \mathrm{~g} / \mathrm{L}$ glucose and supplements as stated above.

After the initial screen a Plackett-Burman design was used to identify key components in the medium affecting proline production and to verify that the nitrogen source significantly affects proline production. The design made it possible to determine the relevant factors with a small number of trials. Twelve factors were screened; all components of CGXII medium and in addition IPTG and arginine. The experimental design and responses are shown in Table 4. Significant effects on proline production were observed when concentrations of glucose, urea, and monopotassium phosphate were varied (Figure 4). A positive effect on proline production was observed for high concentrations of glucose, while low concentrations of urea and monopotassium phosphate improved proline production. As the $t$-values of the effects of glucose, urea, and monopotassium phosphate lie above not only the $t$-value

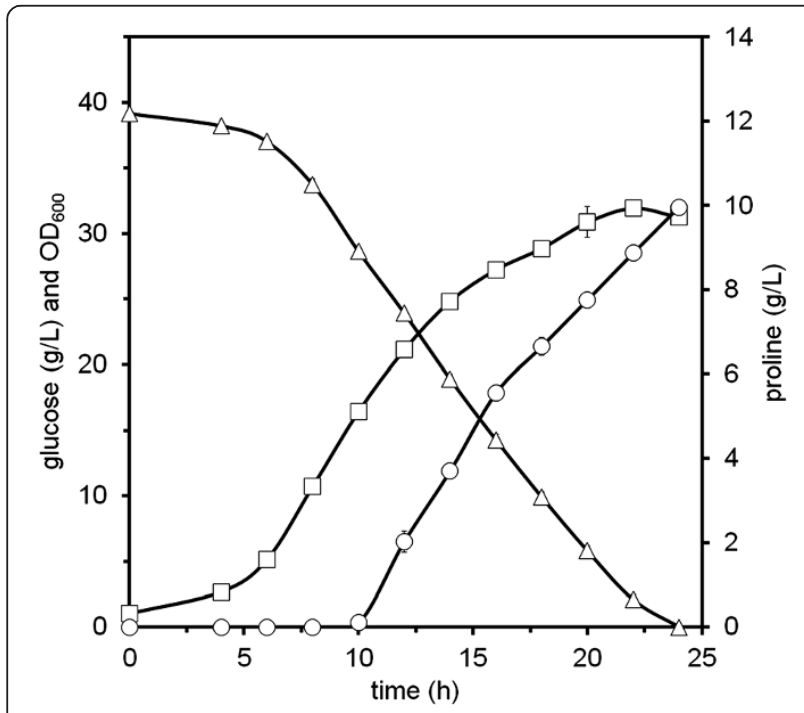

Figure 3 Growth, substrate consumption, and proline formation by C. glutamicum $\mathrm{JJ004}$ in CGXII minimal medium containing $40 \mathrm{~g} / \mathrm{L}$ glucose, $0.75 \mathrm{mM}$ arginine, $1 \mathrm{mM}$ IPTG, and $\mathbf{2 5} \boldsymbol{\mu g} / \mathbf{m L}$ kanamycin. Open square, biomass; open triangle, glucose; open circle, proline. Data are means and standard deviations of at least three cultivations.
Table 3 Proline production with different nitrogen sources

\begin{tabular}{ll}
\hline Nitrogen source & Proline (g/L) \\
\hline Urea, $\left(\mathrm{NH}_{4}\right)_{2} \mathrm{SO}_{4}$ & $8.0 \pm 0.2$ \\
Urea & $11.5 \pm 0.4$ \\
$\left(\mathrm{NH}_{4}\right)_{2} \mathrm{SO}_{4}$ & $6.2 \pm 0.1$ \\
$\mathrm{NH}_{4} \mathrm{Cl}$ & $6.0 \pm 0.1$ \\
\hline
\end{tabular}

Titers were obtained from cells grown in CGXII medium with $40 \mathrm{~g} / \mathrm{L}$ glucose, $0.75 \mathrm{mM}$ arginine, $1 \mathrm{mM}$ IPTG, and $25 \mu \mathrm{g} / \mathrm{mL}$ kanamycin.

threshold, but also the conservative Bonferroni threshold, the components are more likely to be key medium components and their effect not stochastic.

Thus, three different concentrations of urea $(2.5,5$ and $7.5 \mathrm{~g} / \mathrm{L})$, glucose $(20,35$, and $50 \mathrm{~g} / \mathrm{L})$ and monopotassium phosphate $(0.5,1.25$, and $2 \mathrm{~g} / \mathrm{L}$ ) were tested (data not shown) and an improved medium was derived ( $5 \mathrm{~g} / \mathrm{L}$ urea as nitrogen source, $35 \mathrm{~g} / \mathrm{L}$ glucose as carbon source and $2 \mathrm{~g} / \mathrm{L}$ potassium phosphate) and was used for further proline production experiments. Fermentations of JJ004 in the modified medium increased proline production by $25 \%$ compared to production in CGXII medium ( $0.31 \pm 0.01$ as compared to $0.25 \pm 0.003$ g proline/ g glucose, Table 2) and ornithine accumulation was reduced.

\section{Coupling growth to proline production}

In $C$. glutamicum the second enzyme of the ornithine pathway NAGK is feedback inhibited by arginine. The feedback inhibition therefore constitutes a rate-limiting step at high arginine concentrations. Hence, both the feedback alleviation of NAGK and leaky expression of $\operatorname{argF}$ have been employed as means to increase/ couple growth to production [20,23]. Therefore, a feedback alleviated NAGK was overproduced in JJ004 and the resulting strain JJ006 was assayed for NAGK activity. After induction by $1 \mathrm{mM}$ IPTG and growth in BHI complex medium crude extracts of strain JJ006 showed $94 \pm 7$ $\mathrm{mU} / \mathrm{mg}$ specific NAGK activity, which was three fold higher than in the respective control stain JJ005 (30 \pm $7 \mathrm{mU} / \mathrm{mg}$; data not shown). Overproduction of feedback alleviated NAGK entailed increased proline production and reduced by-product formation (Figure 5, Table 5). Less biomass was formed and the growth rate was reduced, however, proline production started early during growth.

\section{Discussion}

C. glutamicum is, especially with regards to carbon and amino acid metabolism, a well-studied bacterium. Nevertheless, details on the proline degradation pathway and regulation of the proline and arginine biosynthetic pathways remain to be elucidated. C. glutamicum can utilise several amino acids as sole carbon and/or nitrogen source 
Table 4 Plackett-Burman design represented by coded values, and proline concentration as the response

\begin{tabular}{|c|c|c|c|c|c|c|c|c|c|c|c|c|c|c|c|c|c|c|c|c|}
\hline Run & A & B & $C$ & D & $E$ & $F$ & $\mathbf{G}$ & $\mathbf{H}$ & 1 & $J$ & $\mathrm{~K}$ & $\mathrm{~L}$ & D1 & D2 & D3 & D4 & D5 & D6 & D7 & $\begin{array}{l}\text { Response } \\
\text { Proline (g/L) }\end{array}$ \\
\hline 1 & - & - & + & + & + & + & - & + & - & + & - & - & - & - & + & + & + & + & + & $4.3 \pm 0.01$ \\
\hline 2 & - & + & - & - & - & - & + & + & - & + & + & - & - & + & + & + & + & - & + & $3.4 \pm 0.05$ \\
\hline 3 & + & + & + & - & + & - & + & - & - & - & - & + & + & - & + & + & + & - & + & $4.1 \pm 0.4$ \\
\hline 4 & + & - & + & - & + & - & - & - & - & + & + & - & + & + & - & - & - & + & + & $5.8 \pm 0.2$ \\
\hline 5 & + & - & - & + & + & + & + & - & + & - & + & - & - & - & - & + & + & - & + & $7.9 \pm 0.3$ \\
\hline 6 & + & - & - & - & - & + & + & - & + & + & - & - & + & + & + & + & + & + & - & $8.2 \pm 0.08$ \\
\hline 7 & - & - & - & - & + & + & - & + & + & - & - & + & + & + & + & - & - & - & + & $5.3 \pm 0.1$ \\
\hline 8 & - & - & + & + & - & + & + & - & - & + & + & + & + & - & + & - & - & - & - & $4.9 \pm 0.01$ \\
\hline 9 & + & - & + & + & - & - & + & + & + & + & - & + & - & + & - & - & - & - & + & $6.7 \pm 0.1$ \\
\hline 10 & + & + & + & + & - & + & - & + & - & - & - & - & + & + & - & + & + & - & - & $5.9 \pm 0.05$ \\
\hline 11 & + & + & - & + & + & - & - & + & + & + & + & - & + & - & + & - & - & - & - & $6.8 \pm 0.08$ \\
\hline 12 & + & + & - & - & + & + & + & + & - & + & - & + & - & - & - & - & - & + & - & $7.7 \pm 0.4$ \\
\hline 13 & - & - & - & - & - & - & - & - & - & - & - & - & - & - & - & - & - & - & - & $6.2 \pm 0$ \\
\hline 14 & + & + & - & + & - & + & - & - & - & - & + & + & - & + & + & - & - & + & + & $7.8 \pm 0.4$ \\
\hline 15 & - & + & + & + & + & - & + & - & + & - & - & - & - & + & + & - & - & + & - & $3.0 \pm 0.2$ \\
\hline 16 & + & - & + & - & - & - & - & + & + & - & + & + & - & - & + & + & + & + & - & $6.5 \pm 0.2$ \\
\hline 17 & - & + & + & - & + & + & - & - & + & + & + & + & - & + & - & + & + & - & - & $2.5 \pm 0.2$ \\
\hline 18 & - & + & - & + & - & - & - & - & + & + & - & + & + & - & - & + & + & + & + & $3.0 \pm 0.2$ \\
\hline 19 & - & + & + & - & - & + & + & + & + & - & + & - & + & - & - & - & - & + & + & $2.7 \pm 0.07$ \\
\hline 20 & - & - & - & + & + & - & + & + & - & - & + & + & + & + & - & + & + & + & - & $5.1 \pm 0.09$ \\
\hline
\end{tabular}

+ indicates that the medium component was used at its high level concentration.

- indicates that the medium component was used at its low level concentration.
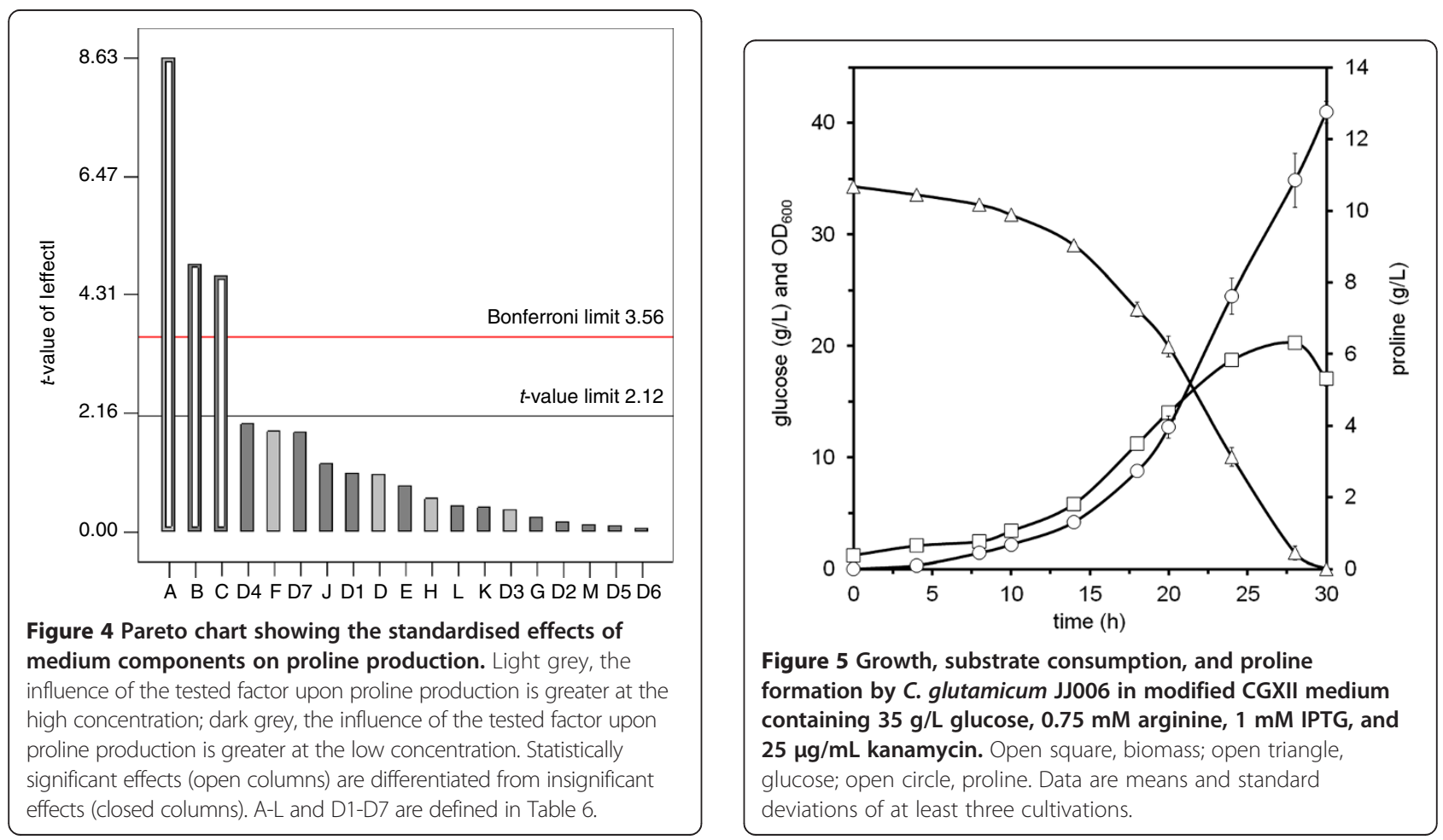
Table 5 Accumulation of proline and byproducts

\begin{tabular}{llllll}
\hline $\begin{array}{l}\text { Proline } \\
(\mathbf{g} / \mathbf{L})\end{array}$ & $\begin{array}{l}\text { Ornithine } \\
(\mathbf{g} / \mathbf{L})\end{array}$ & $\begin{array}{l}\text { Threonine } \\
(\mathbf{g} / \mathbf{L})\end{array}$ & $\begin{array}{l}\text { Alanine } \\
(\mathbf{g} / \mathbf{L})\end{array}$ & $\begin{array}{l}\text { Valine } \\
(\mathbf{g} / \mathbf{L})\end{array}$ \\
\hline$J J 005$ & $11.7 \pm 0.1$ & $<10^{-4}$ & $0.2 \pm 0.005$ & $0.09 \pm 0.005$ & $0.28 \pm 0.004$ \\
$\mathrm{JJ} 006$ & $12.7 \pm 0.3$ & $<10^{-4}$ & $0.09 \pm 0.003$ & $0.09 \pm 0.002$ & $0.16 \pm 0.001$ \\
\hline
\end{tabular}

Titers were obtained from cells grown in CGXIIm with $35 \mathrm{~g} / \mathrm{L}$ glucose, $0.75 \mathrm{mM}$ arginine, $1 \mathrm{mM} \mathrm{IPTG}, 25 \mu \mathrm{g} / \mathrm{mL}$ kanamycin, and $50 \mu \mathrm{g} / \mathrm{mL}$ spectinomycin.

[7] e.g. glutamine has been demonstrated to be an excellent nitrogen source and also allows growth when used as sole carbon and nitrogen source [26]. In the case of proline contradictory observations of its utilisation as nitrogen source have been made $[7,8]$. Bott \& Niebisch reported that C. glutamicum could utilize proline as carbon and nitrogen source, however very slowly. In this study, it was shown that proline did not serve as sole source of carbon for growth of C. glutamicum, and that it is a very poor source of nitrogen.

When comparing proline synthesis starting from 2oxoglutarate via the proline pathway to the conversion via the ornithine pathway, there is a difference in the requirement for ammonia. In the proline biosynthetic pathway one molecule of ammonium is assimilated in the reductive amination of 2-oxoglutarate to glutamate by glutamate dehydrogenase (GDH) per molecule of proline produced (Figure 1). In the OCD-based pathway, a second ammonium molecule is required to be assimilated by GDH, however, it is released during the final ornithine cyclodeaminase reaction. Therefore the conversion of 2-oxoglutarate to glutamate via GDH must be twice as high in the OCD-based pathway as compared to the proline pathway. By medium optimisation employing the Plackett-Burman design it could be shown that a low urea concentration had a positive effect on proline accumulation. While urea utilisation needs to be induced, ammonium assimilation via GDH only shows a weak dependency on nitrogen availability [27]. Besides the low affinity assimilation to ammonium via GDH, C. glutamicum also possesses the high affinity GSGOGAT system for ammonium assimilation which is induced upon nitrogen starvation [27]. The in vivo fluxes of ammonia assimilation via GDH and GS-GOGAT could be determined in C. glutamicum ATCC 13032 and a direct dependency of flux via the GS-GOGAT system on ammonium availability was observed [27]. Under C-limited conditions in a continous culture GS and GOGAT activities in crude extracts were significantly reduced [27]. Proline production was performed with sufficient ammonium, therefore it is assumed that GDH primarily contributes to ammonium assimilation under these conditions.

The genome of C. glutamicum contains a gene annotated to encode ornithine cyclodeaminase [16] within the nitrogen-regulated putative amt-ocd-sox operon [28].
Overexpression of this operon was beneficial for lysine production, but the molecular mechanism remained unknown [29]. The deletion of ocd in an $\operatorname{argF}{ }^{-}$, $\operatorname{argR}^{-}$strain of C. glutamicum increased ornithine production by this strain. Supplementing $5 \mathrm{mM}$ proline improved ornithine production further, which was hypothesized to indicate a possible role of OCD in the conversion of proline to ornithine [30]. As shown here, overexpression of ocd from C. glutamicum neither entailed proline production nor detectable OCD activity, although a SDS-PAGE of crude extracts revealed overproduction of the protein. Thus, the protein encoded by $o c d_{C g}$ either does not possess OCD activity or its activity was too low to be detected. It is interesting to note that multiple protein sequence alignments of biochemically characterised OCDs with putative OCDs [24,31] revealed that some conserved active site residues of OCDs are not conserved in $\mathrm{OCD}_{C g}$. Instead of Arg45 (numbering according to OCD from P. putida), one of three residues whose side chains interact with the ornithine carboxyl group, $\mathrm{OCD}_{\mathrm{Cg}}$ contains a glutamate residue and Asp228, whose side chain forms a hydrogen bond with the leaving ammonia group, is a glycine residue in $\mathrm{OCD}_{C g}$. The lack of conservation of these and further amino acids might explain why no OCD activity could be detected in C. glutamicum. Physiologically, C. glutamicum differs from pseudomonads that typically are able to catabolise arginine and ornithine as sole carbon and nitrogen source. In most Pseudomonas species utilisation of ornithine as carbon source involves succinylation of ornithine, however $P$. putida, which is devoid of such activity, catabolises ornithine via OCD and subsequently via proline degradation [32]. OCD is also involved in opine degradation by Agrobacterium tumefaciens with e.g. the nopaline catabolism region of Ti plasmid C58 encoding OCD for degradation of nopaline via arginine and ornithine to proline [33]. The observation that C. glutamicum does not appear to be able to utilise proline, as shown in this study, or ornithine (unpublished observation) as sole nitrogen or carbon source is commensurate with the lack of OCD activity. It remains to be shown if the protein annotated as putative OCD is active as ornithine cyclodeaminase, or whether it catalyses another reaction.

Heterologous expression of $o c d_{P p}$ by the ORN1 strain resulted in proline accumulation, and a significant increase in production could be achieved by changing the stop codon from TGA to TAA. While examples of modulating translation initiation by changing the start codon or by changing the sequence or spacing of the ribosome binding site exists for C. glutamicum [23], modulating translation termination by altering the stop codon has to the best of our knowledge not yet been reported. Increased OCD levels and activities as consequence of changing the stop codon from TGA to TAA is in line with a bioinformatic 
study on codon usage of C. glutamicum. Putative highly expressed genes exhibited a strong bias for the UAA stop codon, while such a preference was not observed in lowly expressed genes [34]. It is likely that optimisation of sense codons of $o c d_{P p}$ to fit the sense codon preference of C. glutamicum better, could contribute to a further increase in proline production.

As previously demonstrated, glutamate is not limiting the flux through the ornithine pathway, rather it is the feedback inhibition of NAGK by arginine and potentially the feedback inhibition of OAT by ornithine [18,21]. Accordingly, overproduction of feedback-alleviated NAGK improved proline production. In addition, growth was affected as less biomass formed and as the growth rate was reduced. It is noteworthy that proline production already started early during growth which may be beneficial for the overall space-time yield of the process. Moreover, formation of the by-products valine and threonine that are not amino acids of the glutamate family was reduced. As trace amounts of glutamate could be detected in the samples taken for all strains constructed (JJ001JJ006), this is an indication of that the bottleneck in proline production is located between glutamate and proline. A further improvement of the conversion of glutamate to ornithine can be envisioned by alleviating a potential feedback inhibition of OAT and/or employing a bifunctional enzyme with OAT and NAGS activities.

\section{Conclusions}

Heterologous overexpression of ocd in C. glutamicum ORN1 resulted in the overproduction of proline through the ornithine pathway. C. glutamicum JJ004 had a yield of $0.31 \pm 0.01 \mathrm{~g}$ proline/g glucose. Alleviating feedback inhibition of $\mathrm{N}$-acetylglutamate kinase entailed growthcoupled and improved proline production with a yield of $0.36 \pm 0.01 \mathrm{~g} / \mathrm{g}$. The addition of proline to the product palette of the ornithine producing strain ORN1 emphasises that this strain might be exploited as platform strain for industrially relevant bioproducts such as ornithine, proline, putrescine, spermidine, citrulline, and arginine. Moreover, engineering strategies of the platform strain can easily be transferred and applied to improve derived producer strains.

\section{Methods}

\section{Strains, plasmids, and media}

C. glutamicum strain ATCC 13032 [35], ORN1 [21], and its derivatives have been used in this study. As ornithine is a precursor of several interesting products, such as amino acids, di- and polyamines of the glutamate family, ORN1 has the potential to serve as a platform strain. E. coli $\mathrm{DH} 5 \alpha$ [36] was used for the cloning procedures and cultured at $37^{\circ} \mathrm{C}$ in Lysogeny Broth (LB) [37] or on LBagar. Competent E. coli cells and molecular techniques were performed according to standard procedures [37]. Chromosomal DNA from C. glutamicum and Pseudomonas putida KT2440 was isolated by resuspending overnight cultures in $360 \mu \mathrm{L} 50 \mathrm{mM}$ Tris- $\mathrm{HCl}$ (pH 8) followed by the addition of a spatula tip of lysozyme, and incubation at $37^{\circ} \mathrm{C}$ for two hours. Thereafter the procedure "DNA purification from tissues" with the QiaAmp DNA mini kit (Qiagen, Hilden, Germany) was followed. Preparation and transformation of C. glutamicum competent cells was performed according to published methods [38]. Plasmids pVWEx1-ocd $d_{P p 2}$ and pVWEx1-ocd $d_{P p 1}$ were constructed by amplifying ocd from P. putida [NCBI-GeneID: 1046312] with primers ocd2-FW (CTTctgcagAAGGAGATATAG ATATGACGTATTTCATTGATGTTCCA) and ocd3-RV (CCTggtaccTTAGGCAACCCGTCGGATAC, the stop codon was modified from TGA to TAA) or ocd2-RV (CCTggtaccTCAGGCAACCCGTCGGATAC). The amplified fragments were treated with $K p n \mathrm{I}$ and $P s t \mathrm{I}$ and ligated with similarly treated pVWEx1. Plasmid pVWEx1-ocd ${ }_{C g}$ was constructed similarly, however primers ocd1-FW (CTTctgcagAAGGAGATATAGATATGACCGCAACCTA CACCACTG) and ocd1-RV (CCTggtaccTCAAGCCAG TGCGGGTG) were used for the amplification of ocd from C. glutamicum [NCBI-GeneID: 3343467]. The construction of pEKEx3-argB $B_{\mathrm{A} 49 \mathrm{VM} 54 \mathrm{~V}}$ has been described elsewhere [20]. Plasmids pVWEx1, pVWEx1ocd $_{C g}$, pVWEx1-ocd $d_{P p 2}$, pVWEx1-ocd ${ }_{P p 1}$, pEKEx3, and pEKEx3- $\arg B_{\mathrm{A} 49 \mathrm{VM} 54 \mathrm{~V}}$ were transformed into ORN1 resulting in strains JJ001, JJ002, JJ003, JJ004, JJ005, and JJ006, respectively. Brain heart infusion broth (BHI, Roth Chemie GmbH, Karlsruhe, Germany) was used for inoculation of precultures, while CGXII minimal medium $40 \mathrm{~g} / \mathrm{L}$ glucose or CGXIIm (CGXII but without ammonium sulfate) $35 \mathrm{~g} / \mathrm{L}$ glucose was used for growth and proline production.

\section{Culture conditions}

C. glutamicum was inoculated to an OD of 1 in $50 \mathrm{~mL}$ minimal medium, $0.75 \mathrm{mM}$ arginine, $1 \mathrm{mM}$ Isopropyl$\beta$-D-thiogalactopyranosid (IPTG), $25 \mu \mathrm{g} / \mathrm{mL}$ kanamycin, and when required $50 \mu \mathrm{g} / \mathrm{mL}$ spectinomycin on a rotary shaker $(120 \mathrm{rpm})$ in baffled shake flasks at $30^{\circ} \mathrm{C}$. Cultivations were always performed in triplicates. Growth was monitored measuring the OD at $600 \mathrm{~nm}$ using a spectrophotometer (V-1200, VWR, Radnor, PA, USA). 5 g/L urea and $\mathrm{N}$-equimolar concentrations of ammonium sulfate and ammonium chloride were used for screening of nitrogen sources. For the screening of nitrogen sources and the Plackett-Burman design, cells were grown in 48-well flower plates using the Biolector microfermentation system (m2plabs GmbH, Aachen, Germany). $1 \mathrm{~mL}$ medium was used per well with a shaking frequency of $1100 \mathrm{rpm}$. Biomass formation was measured as backscattered light intensity sent at $620 \mathrm{~nm}$ with a signal gain factor of 20 . 
Utilisation of proline as carbon and nitrogen source C. glutamicum was inoculated to an OD of 1 in variants of CGXII medium. For the utilisation of proline as carbon source CGXII with $20 \mathrm{~g} / \mathrm{L}$ glucose or with a C-equimolar concentration of proline was used. For the utilization of proline as nitrogen source CGXII with $5 \mathrm{~g} / \mathrm{L}$ urea and $20 \mathrm{~g} / \mathrm{L}$ ammonium sulfate, with a $\mathrm{N}$-equimolar concentration of proline, or with no nitrogen source added, was used.

\section{Screening of medium components using the Plackett-Burman design}

A Plackett-Burman design [39] for 19 factors (including seven dummies) with 20 runs was employed to screen for factors that significantly affect proline production through the ornithine biosynthetic pathway. The dummies serve as a measure for the error in estimating the main effects. The medium components were screened at a low (-) and a high (+) level, where the concentrations can be found in Table 6 and the design in Table 4. The concentrations of the two levels were selected based on a literature search and preliminary results. The effect of each factor on proline production was determined by the equation:

$$
E\left(X_{i}\right)=2\left(\sum Y_{+i}-\Sigma Y_{-i}\right) / \mathrm{N}
$$

where $E\left(X_{i}\right)$ is the factor main effect, $Y_{+i}$ and $Y_{-i}$ are the proline concentrations in which the factors being tested are at their high and low levels respectively, $\mathrm{N}$ is the number of runs. The $t$-values of the factor main effects were plotted in a Pareto chart, and evaluated based on a $t$-value and a Bonferroni limit [40]. The t-test assesses the risk of declaring an effect significant, when it actually

Table 6 Concentration levels used in the Plackett-Burman experiment

\begin{tabular}{|c|c|c|c|}
\hline \multirow[t]{2}{*}{ Factor } & \multirow[t]{2}{*}{ Name } & \multicolumn{2}{|l|}{ Level } \\
\hline & & - & + \\
\hline$A(g / L)$ & Glucose & 20 & 40 \\
\hline$B(g / L)$ & Urea & 2.5 & 10 \\
\hline$C(g / L)$ & $\mathrm{KH}_{2} \mathrm{PO}_{4}$ & 0.5 & 2.0 \\
\hline$D(g / L)$ & $\mathrm{K}_{2} \mathrm{HPO}_{4}$ & 0.5 & 2.0 \\
\hline$E(g / L)$ & $\mathrm{CaCl}_{2}$ & $5.0 \cdot 10^{-3}$ & $2.0 \cdot 10^{-2}$ \\
\hline$F(g / L)$ & $\mathrm{MgSO}_{4}$ & $1.3 \cdot 10^{-1}$ & $5.0 \cdot 10^{-1}$ \\
\hline $\mathrm{G}(\mathrm{g} / \mathrm{L})$ & MOPS & 31 & 52 \\
\hline$H(m L / L)$ & Trace metals & $7.5 \cdot 10^{-1}$ & 2.0 \\
\hline I $(g / L)$ & Biotin & $1.0 \cdot 10^{-4}$ & $4.0 \cdot 10^{-4}$ \\
\hline$J(g / L)$ & Prochatechuic acid & $1.5 \cdot 10^{-2}$ & $6.0 \cdot 10^{-1}$ \\
\hline$K(g / L)$ & IPTG & $1.2 \cdot 10^{-1}$ & $3.6 \cdot 10^{-1}$ \\
\hline$L(g / L)$ & Arginine & $1.3 \cdot 10^{-1}$ & $1.7 \cdot 10^{-1}$ \\
\hline D1-D7 & Dummy & 0 & 0 \\
\hline
\end{tabular}

was caused by chance. The Bonferroni correction is more conservative taking the number of estimated effects into account by division by the desired probability for the risk value. Effects above the Bonferroni limit are likely not stochastic [40]. The experiment was designed, and obtained data analysed, using the software Design-Expert 8.0.7.1 (Stat-Ease Inc., Minneapolis, USA). The experiment was carried out in duplicates, where the mean was considered the response (Table 4).

\section{Ornithine cyclodeaminase assay}

BHI broth supplemented with $25 \mu \mathrm{g} / \mathrm{mL}$ kanamycin and $1 \mathrm{mM}$ IPTG was inoculated to an OD of 1 and grown for $4 \mathrm{~h}$ at $30^{\circ} \mathrm{C}$. Cells were harvested and washed in 20 $\mathrm{mM} \mathrm{KH} \mathrm{PO}_{4}$ (pH 8.2). Then, cells were lysed by means of sonication (Ultraschalldesintegrator Sonoplus GM 200, Sonotrode M72, Bandelin electronic GmbH \& Co KG, Berlin, Germany) for $6 \mathrm{~min}$ (cycle 0.5, amplitude 55) and centrifuged for $60 \mathrm{~min}$ at $4^{\circ} \mathrm{C}$ and $14600 \mathrm{rpm}$.

Crude extracts were purified using PD10 desalting columns (GE Healthcare, Chalfont St Giles, United Kingdom)

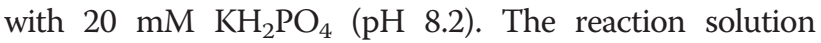
consisted of $20 \mathrm{mM} \mathrm{KH}_{2} \mathrm{PO}_{4}(\mathrm{pH} 8.2)$ and $0.5 \mathrm{mM} \mathrm{NAD}^{+}$. Tubes with reaction solution and between 0.08 and $0.4 \mathrm{mg}$ protein per $250 \mu \mathrm{L}$ reaction were equilibrated to $30^{\circ} \mathrm{C}$ for 3 min in a water bath. The reaction was initiated upon the addition of $25 \mathrm{mM}$ L-ornithine. The reaction was stopped upon addition of $50 \%$ formic acid. The samples were then neutralized with $10 \mathrm{~N} \mathrm{KOH}$ and precipitate was pelleted by centrifugation. Reactions were performed in triplicates with two enzyme concentrations. The conversion of ornithine to proline was measured by HPLC. Unpurified extracts were analysed by SDS-PAGE, and protein quantification was performed by the procedure of Bradford with bovine serum albumin as the standard [41].

\section{$\mathrm{N}$-acetylglutamate kinase assay}

Crude extracts were prepared as stated for the ornithine cyclodeaminase assay. The NAGK activity assay was performed as described by Haas and Leisinger [42]. The assay was performed in triplicates and carried out at $30^{\circ} \mathrm{C}$ at $\mathrm{pH}$ 7.2. One enzyme unit is the amount of enzyme that catalyses the formation of $1 \mu \mathrm{mol}$ of product in $1 \mathrm{~min}$.

\section{Amino acid and glucose determination}

Extracellular amino acids and carbohydrates were quantified by means of high-pressure liquid chromatography (1200 series, Agilent Technologies Deutschland GmbH, Böblingen, Germany). Samples were withdrawn from cultures, centrifuged $(13,000 \times g, 10 \mathrm{~min})$, and the supernatant was used for analysis. For the detection of amino acids, samples were derivatised with 9-fluorenylmethyl chloroformate (FMOC) or ortho-phthaldialdehyde, separated on a system consisting of a pre-column (LiChrospher 
100 RP18 EC-5 $\mu(40 \times 4 \mathrm{~mm})$, CS-Chromatographie Service $\mathrm{GmbH}$, Langerwehe, Germany) and a main col-

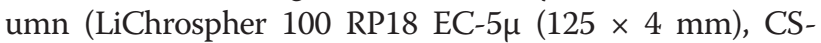
Chromatographie), and detected with a fluorescence detector (FLD G1321A, 1200 series, Agilent Technologies). L-Asparagine was used as internal standard. For the detection of carbohydrates the separation of the analyte was achieved with a column for organic acids $(300 \times$ $8 \mathrm{~mm}, 10 \mu \mathrm{m}$ particle size, $25 \AA$ pore diameter, CSChromatographie) and a refractive index detector (RID G1362A, 1200 series, Agilent Technologies) was used. Derivatisation and quantification was carried out according to published methods [22] with the following modifications of the quantification of FMOC derivatised samples: The mobile phases used were A: $50 \mathrm{mM}$ sodium acetate (pH 4.2) and B: acetonitrile. The gradient used was: 0 min $38 \%$ B, 5 min 38\% B, 12 min 57\% B, 14 min 76\% B, 15 min $76 \% \mathrm{~B}$, and $18 \min 38 \% \mathrm{~B}$.

\section{Competing interests}

The authors declare that they have no competing interests.

\section{Authors' contributions}

VFW and JVKJ designed the experiments. JVKJ conducted the experiments, analysed the results, and wrote the manuscript. VFW reviewed and revised the manuscript. All authors read and approved the final manuscript.

\section{Acknowledgements}

We acknowledge support of the publication fee by Deutsche Forschungsgemeinschaft and the Open Access Publication Funds of Bielefeld University.

Received: 17 May 2013 Accepted: 20 June 2013

Published: 28 June 2013

\section{References}

1. List B: Proline-catalyzed asymmetric reactions. Tetrahedron 2002, 58:5573-5590.

2. Bach TMH, Hara R, Kino K, Ohtsu I, Yoshida N, Takagi H: Microbial production of $\mathrm{N}$-acetyl cis-4-hydroxy-L-proline by coexpression of the Rhizobium L-proline cis-4-hydroxylase and the yeast N-acetyltransferase Mpr1. Appl Microbiol Biotechnol 2013, 97:247-257.

3. Nakanishi T, Yonekura H, Hattori K, Hirao T, Azuma T: Processes for producing L-proline by fermentation. 1989. European Patent EP0098122.

4. Sleator RD, Gahan CGM, Hill C: Mutations in the listerial proB gene leading to proline overproduction: effects on salt tolerance and murine infection. Appl Environ Microbiol 2001, 67:4560-4565.

5. Rönsch H, Krämer R, Morbach S: Impact of osmotic stress on volume regulation, cytoplasmic solute composition and lysine production in Corynebacterium glutamicum MH20-22B. J Biotechnol 2003, 104:87-97.

6. Moxley MA, Becker DF: Rapid reaction kinetics of proline dehydrogenase in the multifunctional proline utilization a protein. Biochemistry 2012, 51:511-520

7. Eggeling L, Reyes O: Nitrogen metabolism and its regulation. In Handbook of Corynebacterium glutamicum. Edited by Eggeling L, Bott M, Boca R. USA: CRC Press; 2005

8. Bott M, Niebisch A: The respiratory chain of Corynebacterium glutamicum. J Biotechnol 2003, 104:129-153.

9. Smith LT: Characterization of a y-glutamyl kinase from Escherichia coli that confers proline overproduction and osmotic tolerance. J Bacteriol 1985, 164:1088-1093.

10. Krishna RV, Leisinger T: Biosynthesis of proline in Pseudomonas aeruginosa. Partial purification and characterization of $\gamma$-glutamyl kinase. Biochem J 1979, 181:215-222.

11. Costilow RN, Laycock L: Reactions involved in the conversion of ornithine to proline in Clostridia. J Bacteriol 1969, 100:622-667.
12. Costilow RN, Laycock L: Ornithine cyclase (deaminating). Purification of a protein that converts ornithine to proline and definition of the optimal assay conditions. J Biol Chem 1971, 246:6655-6660.

13. Dessaux Y, Petit A, Tempe J, Demarez M, Legrain C, Wiame JM: Arginine catabolism in Agrobacterium strains: role of the Ti- plasmid. J Bacteriol 1986, 166:44-50.

14. Leschine SB, Canale-Parola E: Ornithine dissimilation by Treponema denticola. Curr Microbiol 1980, 3:305-310.

15. Stalon V, Vander Wauven C, Momin P, Legrain C: Catabolism of arginine, citrulline and ornithine by Pseudomonas and related bacteria. J Gen Microbiol 1987, 133:2487-2495.

16. Kalinowski J, Bathe B, Bartels D, Bischoff N, Bott M, Burkovski A, Dusch N, Eggeling L, Eikmanns BJ, Gaigalat L, Goesmann A, Hartmann M, Huthmacher K, Krämer R, Linke B, McHardy AC, Meyer F, Möckel B, Pfefferle W, Pühler A, Rey DA, Rückert C, Rupp O, Sahm H, Wendisch VF, Wiegräbe I, Tauch A: The complete Corynebacterium glutamicum ATCC 13032 genome sequence and its impact on the production of L-aspartate-derived amino acids and vitamins. J Biotechnol 2003, 104:5-25.

17. Glansdorff N, Xu Y: Microbial arginine biosynthesis: pathway, regulation and industrial production. In Amino acid biosynthesis - pathways, regulation and metabolic engineering. Edited by Wendisch VF. Berlin: Springer-Verlag; 2007.

18. Sakanyan V, Petrosyan P, Lecocq M, Boyen A, Legrain C, Demarez M, Hallet JN, Glansdorff N: Genes and enzymes of the acetyl cycle of arginine biosynthesis in Corynebacterium glutamicum: enzyme evolution in the early steps of the arginine pathway. Microbiol 1996, 142:99-108.

19. Lee SY, Park JM, Lee JH, Chang ST, Park JS, Kim YH, Min J: Interaction of transcriptional repressor ArgR with transcriptional regulator FarR at the argB promoter region in Corynebacterium glutamicum. App Environ Microbiol 2011, 77:711-718.

20. Schneider J, Niermann K, Wendisch VF: Production of the amino acids L-glutamate, L-lysine, L-ornithine and L-arginine from arabinose by recombinant Corynebacterium glutamicum. J Biotechnol 2010, 154:191-198.

21. Hwang JH, Hwang GH, Cho JY: Effect of increased glutamate availability on L-ornithine production in Corynebacterium glutamicum. J Microbiol Biotechnol 2008, 18:704-710.

22. Schneider J, Wendisch VF: Putrescine production by engineered Corynebacterium glutamicum. Appl Microbiol Biotechnol 2010, 88:859-868.

23. Schneider J, Eberhardt D, Wendisch VF: Improving putrescine production by Corynebacterium glutamicum by fine-tuning ornithine transcarbamoylase activity using a plasmid addiction system. Appl Microbiol Biotechnol 2012, 95:169-178.

24. Goodman JL, Wang S, Alam S, Ruzicka FJ, Frey PA, Wedekind JE: Ornithine cyclodeaminase: structure, mechanism of action, and implications for the mu-crystallin family. Biochem 2004, 43:13883-13891.

25. Peters-Wendisch PG, Schiel B, Wendisch VF, Katsoulidis E, Möckel B, Sahm H, Eikmanns BJ: Pyruvate carboxylase is a major bottleneck for glutamate and lysine production by Corynebacterium glutamicum. J Mol Microbiol Biotechnol 2001, 3:295-300.

26. Rehm N, Georgi T, Hiery E, Degner U, Schmiedl A, Burkovski A, Bott M: L-glutamine as a nitrogen source for Corynebacterium glutamicum: derepression of the AmtR regulon and implications for nitrogen sensing. Microbiol 2010, 156:3180-3193.

27. Tesch M, de Graaf AA, Sahm H: In vivo fluxes in the ammoniumassimilatory pathways in Corynebacterium glutamicum studied by ${ }^{15} \mathrm{~N}$ nuclear magnetic resonance. Appl Environ Microbiol 1999, 65:1099-1109.

28. Jakoby M, Kramer R, Burkovski A: Nitrogen regulation in Corynebacterium glutamicum: isolation of genes involved and biochemical characterization of corresponding proteins. FEMS Microbiol Lett 1999, 173:303-310.

29. Sindelar G, Wendisch VF: Improving lysine production by Corynebacterium glutamicum through DNA microarray-based identification of novel target genes. Appl Microbiol Biotechnol 2007, 76:677-689.

30. Lee SY, Cho JY, Lee HJ, Kim YH, Min J: Enhancement of ornithine production in proline-supplemented Corynebacterium glutamicum by ornithine cyclodeaminase. J Microbiol Biotechnol 2010, 20:127-131.

31. Trovato M, Maras B, Linhares F, Costantino P: The plant oncogene rolD encodes a functional ornithine cyclodeaminase. PNAS 2001, 98:13449-13453.

32. Tricot C, Stalon V, Legrain C: Isolation and characterization of Pseudomonus putida mutants affected in arginine, ornithine and citrulline catabolism: 
function of the arginine oxidase and arginine succinyltransferase pathways. J Gen Microbiol 1991, 137:2911-2918.

33. Sans N, Schröder G, Schröder J: The Noc region of Ti plasmid C58 codes for arginase and ornithine cyclodeaminase. Eur J Biochem 1987, 167:81-87.

34. Liu G, Wu J, Yang H, Bao Q: Codon usage patterns in Corynebacterium glutamicum: mutational bias, natural selection and amino acid conservation. Comp Funct Genomics 2010, 2010:7. 343569.

35. Abe S, Takayama K, Kinoshita S: Taxonomical studies on glutamic acid producing bacteria. J Gen Appl Microbiol 1967, 13:279-301.

36. Hanahan D: Studies on transformation of Escherichia coli with plasmids. J Mol Biol 1983, 166:557-580.

37. Sambrook J, Fitsch EF, Maniatis T: Molecular cloning: a laboratory manual. Cold Spring Harbor: Cold Spring Harbor Press; 1989.

38. Eggeling L, Reyes O: Experiments. In Handbook of Conynebacterium glutamicum. Edited by Eggeling L, Bott M, Boca R. USA: CRC Press; 2005:3535-3566.

39. Plackett RL, Burman JP: The design of optimum multifactorial experiments. Biometrika 1946, 33:305-325.

40. Anderson MJ, Whitcomb PJ: Chapter 3: Two-level factorial design. In DOE simplified: practical tools for effective experimentation. 2nd edition. New York, USA: Productivity Press; 2007.

41. Bradford MM: A rapid and sensitive method for the quantitation of microgram quantities of protein utilizing the principle of protein-dye binding. Anal Biochem 1976, 72:248-254.

42. Haas D, Leisinger T: N-acetylglutamate 5-phosphotransferase of Pseudomonas aeruginosa. Catalytic and regulatory properties. Eur J Biochem 1975, 52:377-383.

doi:10.1186/1475-2859-12-63

Cite this article as: Jensen and Wendisch: Ornithine cyclodeaminasebased proline production by Corynebacterium glutamicum. Microbial Cell Factories 2013 12:63.

\section{Submit your next manuscript to BioMed Central and take full advantage of:}

- Convenient online submission

- Thorough peer review

- No space constraints or color figure charges

- Immediate publication on acceptance

- Inclusion in PubMed, CAS, Scopus and Google Scholar

- Research which is freely available for redistribution 